\title{
Misguided Progression Analysis by Optical Coherence Tomography: A Report of Two Cases
}

\author{
Jeffrey B. Kennedy Jeffrey R. SooHoo Leonard K. Seibold \\ Malik Y. Kahook \\ Department of Ophthalmology, University of Colorado School of Medicine, \\ Aurora, Colo., USA
}

\section{Key Words}

Glaucoma diagnosis - Spectral domain optical coherence tomography · Nerve fibers; pathology · Optic nerve diseases; diagnosis · Guided Progression Analysis

\begin{abstract}
Spectral domain optical coherence tomography (SOCT) is an important tool for the diagnosis and management of glaucoma. In an effort to help automate the detection of progressive structural glaucomatous optic nerve damage, Carl Zeiss Meditec has developed Guided Progression Analysis (GPA) software in conjunction with the Cirrus SOCT (Carl Zeiss Meditec, Dublin, Calif., USA). Here, we report a case in which the GPA merged information from 2 different patients, and another case in which data from alternate eyes was compared, making the final analysis invalid.

(C) 2014 S. Karger AG, Basel
\end{abstract}

\section{Introduction}

Glaucoma is a heterogeneous group of diseases characterized by progressive optic neuropathy. The disease process involves structural changes including thinning of the optic nerve neuroretinal rim and retinal nerve fiber layer (RNFL) and corresponding visual field loss [1]. Glaucomatous structural changes may precede functional visual field loss, as demonstrated by perimetry [2]. The development of spectral domain optical coherence tomography (SOCT) has aided in the detection of progressive thinning of the RNFL $[3,4]$ and has become an integral part of monitoring patients with glaucoma.

In an effort to help automate the process of detecting early or subtle glaucomatous progression, Carl Zeiss Meditec has developed Guided Progression Analysis (GPA) software 
Kennedy et al.: Misguided Progression Analysis by Optical Coherence Tomography: A Report of Two Cases

for use with the Cirrus SOCT (Carl Zeiss Meditec, Dublin, Calif., USA). This software performs both event- and trend-based analysis to detect changes in the RNFL and optic nerve rim. Na et al. [5] have recently demonstrated that GPA might be a useful adjunct in detecting glaucomatous progression. However, the software showed poor agreement with expert analysis of stereo color and red-free optic nerve photos. The following cases illustrate two instances in which the GPA software used the wrong baseline information to generate the event and trend analyses.

\section{Case Presentations}

Case 1

Patient 1 was a 56-year-old female glaucoma suspect who presented for a follow-up examination, which included SOCT scanning. Optic nerve head examination by slit lamp demonstrated mild asymmetric cupping without evidence of focal RNFL thinning. The GPA output of the patient's left eye (fig. 1) included the correct scan from the most recent visit; however, both baseline scans appeared to be that of a right eye instead of a left eye. Neither the scan date nor the RNFL map corresponded to the previous right eye scans from this same patient. The RNFL thickness profile demonstrated a very different contour when the baseline scans were compared to the current scan. A review of the RNFL thickness profile suggested that both baseline scans corresponded to the same patient as they had similar contours. This contour, however, was dissimilar from the patient's current scan. By reviewing all OCT scans performed on the same day as the erroneously imported baseline scans, we were able to identify the patient who corresponded to these data. It appeared that data were retrieved from both the wrong patient and the wrong eye for use in the GPA output of the patient scanned on this visit.

Case 2

Patient 2 was a 68-year-old male with primary open angle glaucoma. The GPA of the right eye (fig. 2) imported a baseline scan of a left eye. On review of previous OCT data, the first baseline scan used in this analysis was determined to be a scan of the correct patient from the correct date. However, the wrong eye was included in the analysis. Because of this erroneously imported baseline scan, the trend analysis of the superior RNFL mistakenly suggested a significant downward slope indicating a loss of $5.2 \mu \mathrm{m} /$ year.

\section{Discussion}

The detection of glaucomatous progression is complex and requires the assimilation of multiple data points to analyze structural changes at the level of the RNFL as well as visual field changes. Technology has evolved to assist clinicians in making decisions regarding the diagnosis and treatment of glaucoma. The GPA software that accompanies the Humphrey Field Analyzer (Carl Zeiss Meditec) is an example of a tool that has shown promise towards automating the detection of glaucomatous visual field progression [6]. With the advent of SOCT and high-resolution imaging of the RNFL, there is an opportunity to provide similar automated statistical analysis to help clinicians diagnose glaucoma and its progression over time. The above cases demonstrated erroneous data analyses due to the utilization of baseline scans from both the wrong eye of the wrong patient and the wrong eye of the correct patient. 
Kennedy et al:: Misguided Progression Analysis by Optical Coherence Tomography: A Report of Two Cases

It is unclear at this point exactly how these errors occurred. These events were reported both to the manufacturer and to the Food and Drug Administration (FDA) Manufacturer and User Device Experience database. At this time, we are unaware of any similar incidents at other institutions.

Although an operator error certainly has to be considered, we were unable to purposely reproduce these anomalous analyses and could not come up with a plausible method for an operator to cause such issues. In both cases, the data erroneously included in the GPA analysis were identified retrospectively based on the time stamp and the RNFL thickness map. In both cases, the original scans were appropriately labeled as the correct eye and with the correct patient identifier. This suggests that an operator labeling error was not the cause of the erroneous inclusion in the GPA.

Carl Zeiss Meditec, after personal communication, also could not envision a scenario where an operator could have caused these errors, pointing instead to a likely software malfunction. Regardless of the cause, it is apparent that errors of this type may compromise patient care. In the cases presented herein, the utilization of incorrect baseline scans was easily detected because an alternate eye was displayed in the RNFL map. If a scan from a different patient but the correct eye were to be included, it would potentially be much more difficult to identify. If the errant scan is not detected and incorrect data points are used for GPA, the software may suggest progression (or not detect actual progression), as illustrated in Case 2 above.

We recommend that all clinicians utilizing GPA software be aware of these potential errors and confirm the appropriate baseline scans are chosen in each analysis. Both the RNFL thickness map and the RNFL thickness profile provide a visual representation of the raw data and can help the clinician identify inappropriately imported data.

\section{Disclosure Statement}

Dr. Kennedy, Dr. SooHoo, and Dr. Kahook have no financial interests to disclose. Dr. Seibold reports grants from Alcon, nonfinancial support from Sensimed, and grants from Bausch and Lomb, all outside the submitted work.

\section{References}

1 Hood D, Kardon R: A framework for comparing structural and functional measures of glaucomatous damage. Prog Retin Eye Res 2007;26:688-710.

2 Medeiros F, Alencar L, Zangwill L, Bowd C, Sample P, Weinreb R: Prediction of functional loss in glaucoma from progressive optic disc damage. Arch Ophthalmol 2009;127:1250-1256.

3 Mwanza J, Oakley J, Budenz D, Anderson D: Ability of cirrus HD-OCT optic nerve head parameters to discriminate normal from glaucomatous eyes. Ophthalmology 2011;118:241-248.

-4 Kotowski J, Wollstein G, Folio L, Ishikawa H, Schuman J: Clinical use of OCT in assessing glaucoma progression. Ophthalmic Surg Lasers Imaging 2011;42:S6-S14.

-5 Na J, Sung K, Baek S, Lee J, Kim S: Progression of retinal nerve fiber layer thinning in glaucoma assessed by cirrus optical coherence tomography-guided progression analysis. Curr Eye Res 2013;38:386-395.

-6 Arnalich-Montiel F, Casas-Llera P, Muñoz-Negrete FJ, Rebolleda G: Performance of glaucoma progression analysis software in a glaucoma population. Graefes Arch Clin Exp Ophthalmol 2009;247:391-397. 
Kennedy et al.: Misguided Progression Analysis by Optical Coherence Tomography: A
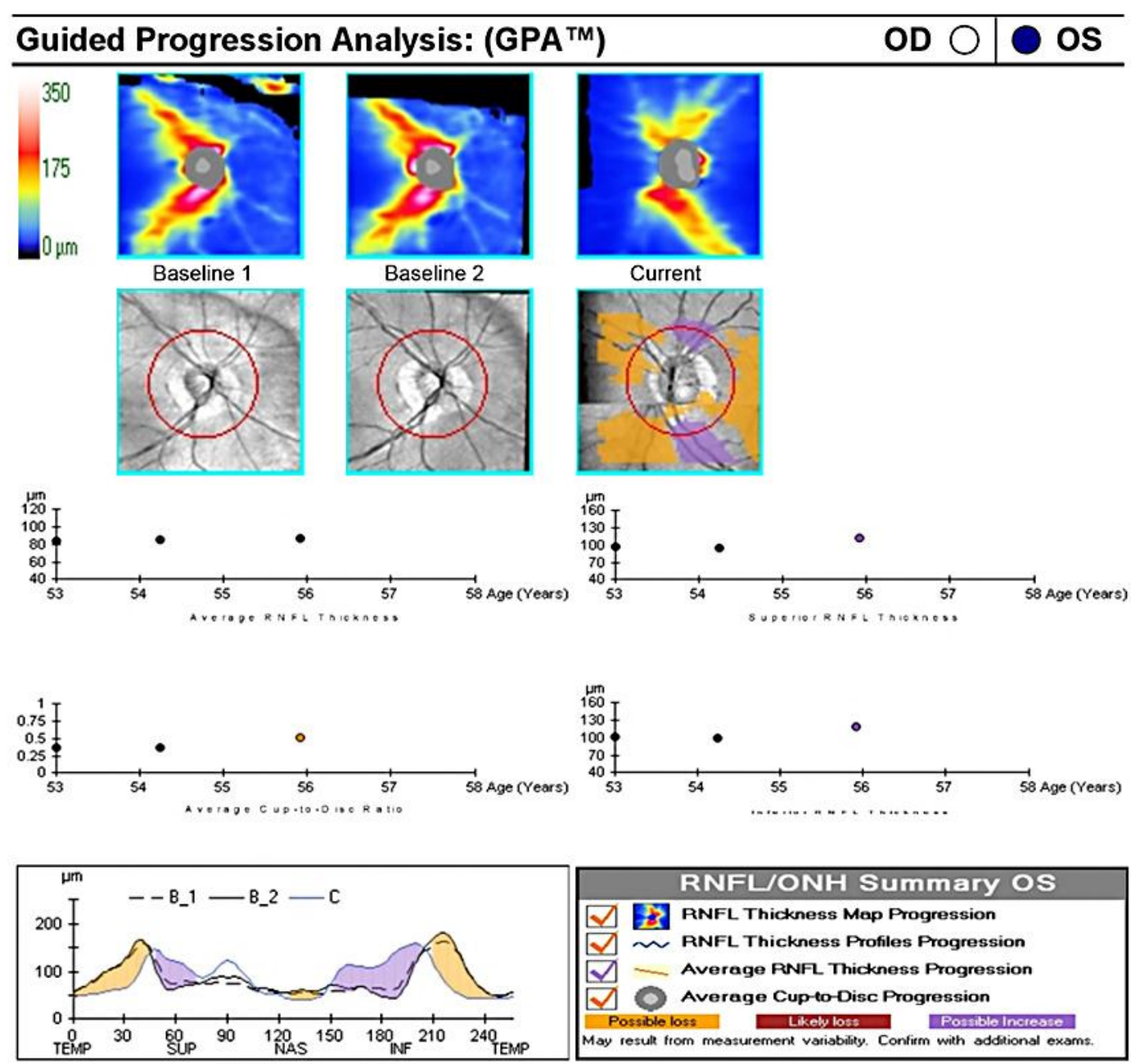

\section{RNFL/ONH Summary OS}

$\checkmark$ FNFL Thickness Map Progression

$\checkmark$ RNFL Thickness Profiles Progression

$\checkmark$ Average PNFL Thickness Progression

$\checkmark$ Average Cup-to-Disc Progression

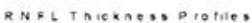

Fig. 1. Left eye GPA for Patient 1. Note that both baseline scans appear to be from a right eye. Additionally, the RNFL thickness maps and profiles from the baseline scans do not correspond to Patient 1's current optical coherence tomography scan. 
Kennedy et al.: Misguided Progression Analysis by Optical Coherence Tomography: A Report of Two Cases

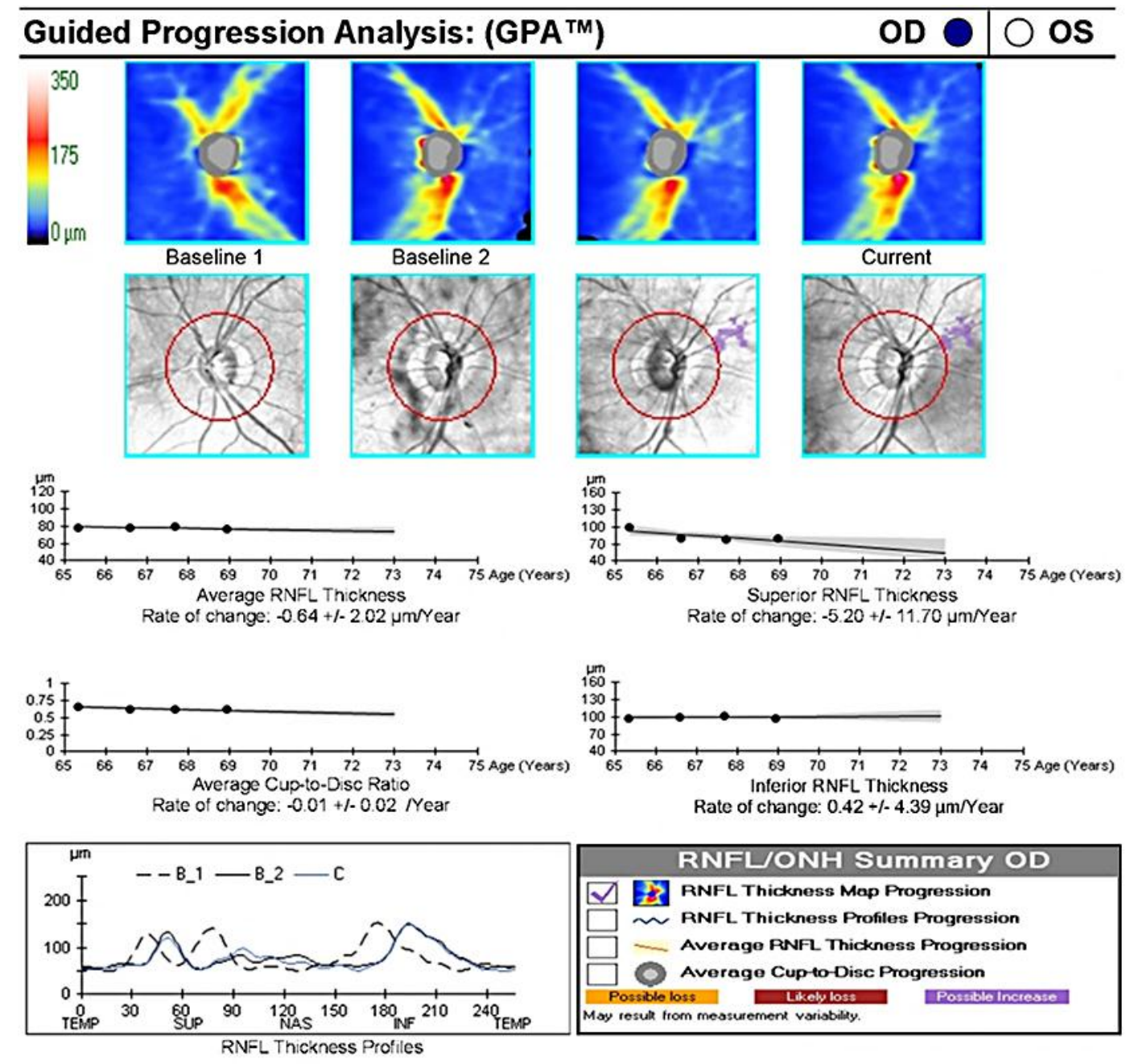

Fig. 2. Right eye GPA for Patient 2. Note that the first baseline scan appears to be a scan of a left eye. 